\title{
Impact of non-invasive follicular thyroid neoplasm with papillary-like nuclear features on the Bethesda system for reporting thyroid cytopathology: a multi-institutional study in five Asian countries
}

\author{
Andrey Bychkov ${ }^{1}$, Somboon Keelawat ${ }^{1}$, Shipra Agarwal ${ }^{2}$, Deepali Jain ${ }^{2}$, \\ Chan Kwon Jung ${ }^{3}$, SoonWon Hong ${ }^{4}$, Chiung-Ru Lai ${ }^{5}$, Shinya Satoh ${ }^{6}$, \\ KeNNICHI KAKUdo ${ }^{7}$ \\ ${ }^{1}$ Department of Pathology, Faculty of Medicine, Chulalongkorn University, Bangkok, \\ Thailand; ${ }^{2}$ Department of Pathology, All India Institute of Medical Sciences, New Delhi, \\ India; ${ }^{3}$ Department of Hospital Pathology, College of Medicine, The Catholic University of \\ Korea, Seoul, Republic of Korea; ${ }^{4}$ Department of Pathology, Yonsei University, College of \\ Medicine, Seoul, Republic of Korea; ${ }^{5}$ Department of Pathology, Taipei Veterans General \\ Hospital, Taipei, Taiwan; ${ }^{6}$ Department of Endocrine Surgery, Yamashita Thyroid Hospital, \\ Fukuoka, Japan; ${ }^{7}$ Department of Pathology, Nara Hospital, Kindai University Faculty of \\ Medicine, Nara, Japan
}

\begin{abstract}
Summary
Several Western studies showed that the recent introduction of non-invasive follicular thyroid neoplasm with papillary-like nuclear features (NIFTP) significantly decreased risk of malignancy for cytological diagnostic categories. We aimed to determine the impact of NIFTP on risk of malignancy within a cohort of thyroid nodules from Asian countries, and to compare distribution of diagnostic categories between NIFTP and invasive encapsulated follicular variant of papillary thyroid carcinoma (eFV-PTC). Consecutive thyroid fineneedle aspirates from six institutions were retrospectively analysed. Histopathology slides with a diagnosis of eFVPTC were reviewed and reclassified into invasive eFV-PTC and NIFTP. The risk of malignancy was calculated with and without NIFTP. Of 11,372 thyroid nodules, 2044 had available surgical follow-up. NIFTP was diagnosed in 59 cases, which constituted $2.9 \%$ of all excised nodules, and $5.3 \%$ of malignant nodules. Preoperative cytological diagnoses for NIFTP were non-diagnostic $(10.2 \%)$, benign (18.6\%), atypia of undetermined significance/follicular lesion of undetermined significance (22.0\%), follicular neoplasm/ suspicious for follicular neoplasm (FN/SFN) (32.2\%), suspicious for malignancy (SM) (11.9\%), and malignant (5.1\%). The only category which showed a relative reduction in risk of malignancy after reclassification of more than $20 \%$, was FN/SFN (24.4\%). There was a significantly higher prevalence of benign cytology in NIFTP $(p=0.04)$ and SM/malignant in invasive eFV-PTC $(p=0.05)$. A majority of NIFTP cases were classified in indeterminate categories, which decreased the corresponding risk of malignancy. However, the magnitude of NIFTP impact was much lower than in the Western reports. Asian countries may not experience significant effects of NIFTP reclassification on the practice of thyroid cytopathology.
\end{abstract}

Key words: Non-invasive follicular thyroid neoplasm with papillary-like nuclear features; NIFTP; the Bethesda system for reporting thyroid cytopathology; fine-needle aspiration (FNA); risk of malignancy (ROM); thyroid nodules; Asia; papillary thyroid carcinoma; follicular variant of papillary thyroid carcinoma.

Received 21 September, revised 30 October, accepted 5 November 2017 Available online 7 April 2018

\section{INTRODUCTION}

Fine-needle aspiration (FNA) biopsy plays a key role in the management of thyroid nodules. The Bethesda System for Reporting Thyroid Cytopathology (TBSRTC) has been widely adopted as a standard for reporting thyroid FNA results. ${ }^{1}$ Each of the six Bethesda categories has implied risk of malignancy and recommended clinical management. A substantial amount of thyroid nodules $(20-25 \%)$ is classified as indeterminate on preoperative FNA, ${ }^{2,3}$ which poses difficulties for the management algorithms. Further surgical excision yields malignancy in up to one-third of indeterminate nodules, ${ }^{3,4}$ being usually represented by the follicular variant of papillary thyroid carcinoma (FV-PTC). Recent studies found that encapsulated FV-PTC (eFV-PTC) without invasion has an excellent prognosis with extremely low risk of adverse outcomes compared to its invasive counterpart. As a tribute to the highly indolent behaviour of noninvasive eFV-PTC and to reduce overtreatment of the patients with this tumour, the term non-invasive follicular thyroid neoplasm with papillary-like nuclear features (NIFTP) was proposed by the international panel of thyroid experts.

The introduction of the new terminology of NIFTP may change current clinical and laboratory practices in several ways, the cornerstone being patients who will not be labelled as having cancer and not overtreated. ${ }^{5}$ Accordingly, medical professionals involved in the management of these patients may need to adjust current approaches and guidelines to the new entity. ${ }^{6}$ All this is expected to have an impact on health 
care with significant savings due to less aggressive and expensive treatment. ${ }^{5}$ NIFTP may significantly influence the practice of endocrine pathology. This involves a change of risk of malignancy in diagnostic cytological categories, which may alter decision making, as well as sampling and evaluation of surgical specimens and performance of molecular tests. ${ }^{5,6}$ Introduction of the new histological entity NIFTP inevitably influences preoperative thyroid FNA. The risk of malignancy for each of the Bethesda categories was initially predicated considering non-invasive eFV-PTC as malignancy. ${ }^{1}$ However, the change in terminology could shift malignancy risk and impact the management algorithm associated with each diagnostic category of TBSRTC.

NIFTP is placed mainly in Bethesda categories III-V. ${ }^{7-9}$ A similar distribution pattern is typical for invasive eFVPTC, which is supposed to progress from NIFTP. 5,9 Several studies demonstrated that introduction of NIFTP terminology affected risk of malignancy for the Bethesda diagnostic categories. ${ }^{7,10-12}$ These individual studies found that if tumours currently designated as NIFTP were considered non-malignant, a significant decrease in risk of malignancy would occur in the indeterminate categories. A recent meta-analysis based on four independent datasets showed that after adjustment to NIFTP terminology, risk of malignancy was significantly lower in the categories II-V. ${ }^{11}$

It is obvious that the magnitude of all changes related to the NIFTP reclassification will be dependent on the prevalence of NIFTP in a given population. ${ }^{6,12}$ Nikiforov et al. reported $18.6 \%$ of NIFTP among all PTC based on data from four American and Italian institutions. ${ }^{5}$ Similar rates ranging from $15 \%$ to $28 \%$ were further reported from the USA, South America, and Europe. ${ }^{7,12,13}$ The Asian continent significantly contributes to the world prevalence of thyroid cancer. GLOBOCAN estimated that $48 \%$ of all new thyroid cancer cases are diagnosed in Asia. ${ }^{14}$ Recently, we collected original data on the NIFTP incidence from six Asian countries and found that NIFTP was rare (mean rate $1.5 \%$ ) in the local practice. $^{15}$

This study aimed to determine the impact of NIFTP on risk of malignancy for the Bethesda categories within a large Asian multi-institutional cohort. Additionally, we intended to validate the rate of NIFTP in the Asian population and to compare the distribution of NIFTP with invasive eFV-PTC in the Bethesda system.

\section{MATERIALS AND METHODS}

The study cohort was collected from the six large-to-mid scale tertiary thyroid cancer centres representing five Asian countries: King Chulalongkorn Memorial Hospital (Bangkok, Thailand), All India Institute of Medical Sciences (New Delhi, India), Taipei Veterans General Hospital (Taipei, Taiwan), Yamashita Thyroid Hospital (Fukuoka, Japan), Gangnam Severance Hospital (Seoul, Korea), and Seoul St Mary's Hospital (Seoul, Korea). A cohort from Thailand has been described previously without any relation to the NIFTP terminology. ${ }^{16}$ Data collection was approved by the institutional review board or its equivalent for individual institutions.

Local databases were searched retrospectively for all consecutive thyroid FNA cases over the applicable time period (1-3 years). Cytological smears from cervical lymph nodes, perithyroid lesions with uncertain location, and consultation cases were excluded. Terminology of TBSRTC has been used. ${ }^{1}$ The diagnostic categories were non-diagnostic (category I), benign (B, category II), atypia of undetermined significance/follicular lesion of undetermined significance (AUS/FLUS, category III), follicular neoplasm/suspicious for follicular neoplasm (FN/SFN, category IV), suspicious for malignancy (SM, category V), and malignant (M, category VI).
Relevant demographic (Table 1) and surgical follow-up data were collected. Cases with subsequent resection were correlated with the nodule location provided in the cytopathology report, and only those matched were included in the study. For cases with repeated FNA, only the latest preoperative cytological diagnosis was considered. Surgical pathology reports were reviewed, and all cases with the diagnosis of FV-PTC were categorised as encapsulated or infiltrative. All slides with the diagnosis of eFV-PTC were retrieved from the archives and reviewed on-site by pathologists with thyroid expertise. These cases were further stratified into invasive eFV-PTC and noninvasive (NIFTP). The latter was applied only under strict diagnostic criteria proposed by Nikiforov et al. ${ }^{5}$ Briefly, to qualify as NIFTP a tumour should exhibit nuclear features of PTC, be encapsulated or circumscribed, show no signs of capsular/vascular invasion, and contain less than $1 \%$ of papillary pattern.

The risk of malignancy expressed in per cent for each Bethesda diagnostic category was determined by dividing the total number of malignant cases by the number of cases with surgical follow-up within particular TBSRTC category. Calculations were performed considering NIFTP as a malignant and non-malignant tumour. The difference between both results constitutes a decrease in risk of malignancy, which was calculated as the absolute and relative decrease in risk of malignancy.

All data were entered into Microsoft Office Excel 2007 spreadsheet software (Microsoft Corporation, USA). All statistical analyses were performed using SPSS Statistics version 17.0 (SPSS IBM, USA). Fisher's exact test was used to compare categorical variables. A $p$ value of less than 0.05 was considered statistically significant.

\section{RESULTS}

The majority of the resected nodules were preoperatively diagnosed as malignant $(34.0 \%)$ and benign $(27.1 \%)$, followed by non-diagnostic (12.0\%), AUS/FLUS (10.5\%), FN/SFN (9.4\%) and SM (6.9\%) categories. The latter three categories traditionally interpreted as indeterminate thyroid nodules $^{17}$ accounted for over one-quarter $(26.8 \%)$ of all samples. Implied risk of malignancy was $21.6 \%, 13.2 \%$, $44.7 \%, 40.4 \%, 88.0 \%$, and $98.7 \%$ for the Bethesda categories I to VI, respectively (Table 2). There was a wide variation of risk of malignancy among institutions (Fig. 1, Table 3). Risk of malignancy for each Bethesda category showed an increasing trend as overall risk of malignancy of all resected nodules increased (Fig. 1).

\section{Risk of malignancy after NIFTP adjustment}

A total of 59 cases were reclassified as NIFTP after review of the slides (Table 2). This was equal to $2.9 \%$ incidence among all excised thyroid nodules, and 5.3\% among all malignancies. NIFTP cases represented the following TBSRTC categories shown in decreasing order of frequency: FN/SFN (32.2\%), AUS/FLUS (22.0\%), benign (18.6\%), SM (11.9\%), non-diagnostic (10.2\%), and malignant (5.1\%). Roughly twothirds of NIFTP cases $(66.1 \%)$ belonged to the indeterminate FNA categories.

Absolute decrease in risk of malignancy after eliminating NIFTP from the malignancy group was comparatively minor, even for FN/SFN and AUS/FLUS categories (Table 2). The relative decrease is a more representative estimate of risk of malignancy alteration. The greatest relative decrease in risk of malignancy (24.4\%) was observed for FN/SFN. None of the other TBSRTC categories, including AUS/FLUS and SM, showed a relative risk of malignancy reduction of more than $20 \%$ (Table 2). Accordingly, the differences in the risk of malignancy before and after NIFTP reclassification were not statistically significant for all the diagnostic categories. Only the 
Table 1 Study demographics

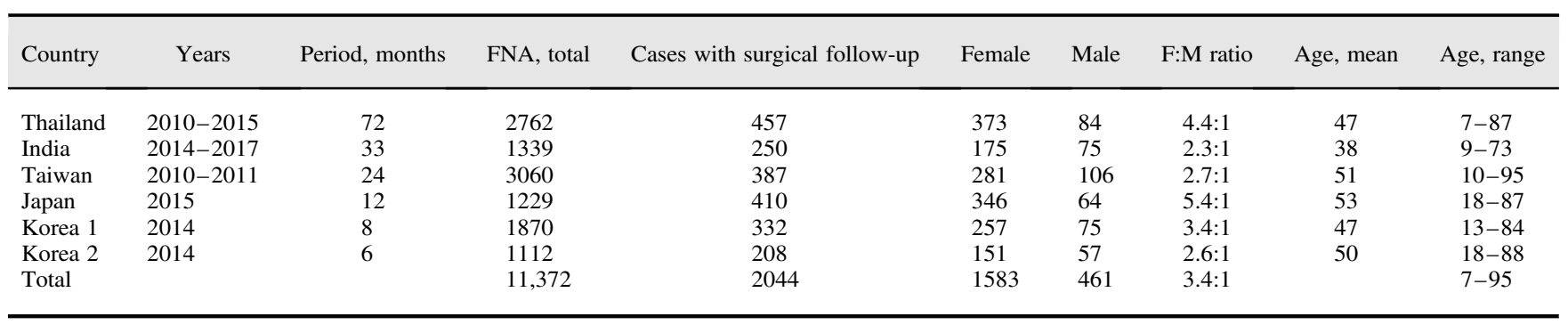

FNA, fine-needle aspiration.

Table 2 Risk of malignancy in the Bethesda diagnostic categories before and after implementation of the NIFTP terminology

\begin{tabular}{|c|c|c|c|c|c|c|}
\hline & \multicolumn{6}{|c|}{ Bethesda system diagnostic category } \\
\hline & I & II & III & IV & $\mathrm{V}$ & VI \\
\hline Number of each Bethesda category (A) & 2772 & 6219 & 766 & 374 & 231 & 1010 \\
\hline Cases with surgical follow-up (B) & 245 & 554 & 215 & 193 & 142 & 695 \\
\hline Surgical resection rate $(\mathrm{B} / \mathrm{A})$ & $8.8 \%$ & $8.9 \%$ & $28.1 \%$ & $51.6 \%$ & $61.5 \%$ & $68.8 \%$ \\
\hline Malignancy on surgery $(\mathrm{C})$ & 53 & 73 & 96 & 78 & 125 & 686 \\
\hline ROM among all patients in each Bethesda category $(\mathrm{C} / \mathrm{A})^{\mathrm{a}}$ & $1.9 \%$ & $1.2 \%$ & $12.5 \%$ & $20.9 \%$ & $54.1 \%$ & $67.9 \%$ \\
\hline Actual ROM in nodules surgically excised $(\mathrm{C} / \mathrm{B})$ & $21.6 \%$ & $13.2 \%$ & $44.7 \%$ & $40.4 \%$ & $88.0 \%$ & $98.7 \%$ \\
\hline NIFTP (D) & 6 & 11 & 13 & 19 & 7 & 3 \\
\hline Actual ROM without NIFTP [(C-D)/B] & $19.2 \%$ & $11.2 \%$ & $38.6 \%$ & $30.6 \%$ & $83.1 \%$ & $98.3 \%$ \\
\hline Absolute decrease in ROM & $2.4 \%$ & $2.0 \%$ & $6.0 \%$ & $9.8 \%$ & $4.9 \%$ & $0.4 \%$ \\
\hline Relative decrease in ROM & $11.3 \%$ & $15.1 \%$ & $13.5 \%$ & $24.4 \%$ & $5.6 \%$ & $0.4 \%$ \\
\hline
\end{tabular}

NIFTP, non-invasive follicular thyroid neoplasm with papillary-like nuclear features; ROM, risk of malignancy.

${ }^{a}$ This represents the lower-bound estimate of the true prevalence of malignancy for each Bethesda category as not all patients undergo surgical resection of the thyroid nodule and histological diagnosis.
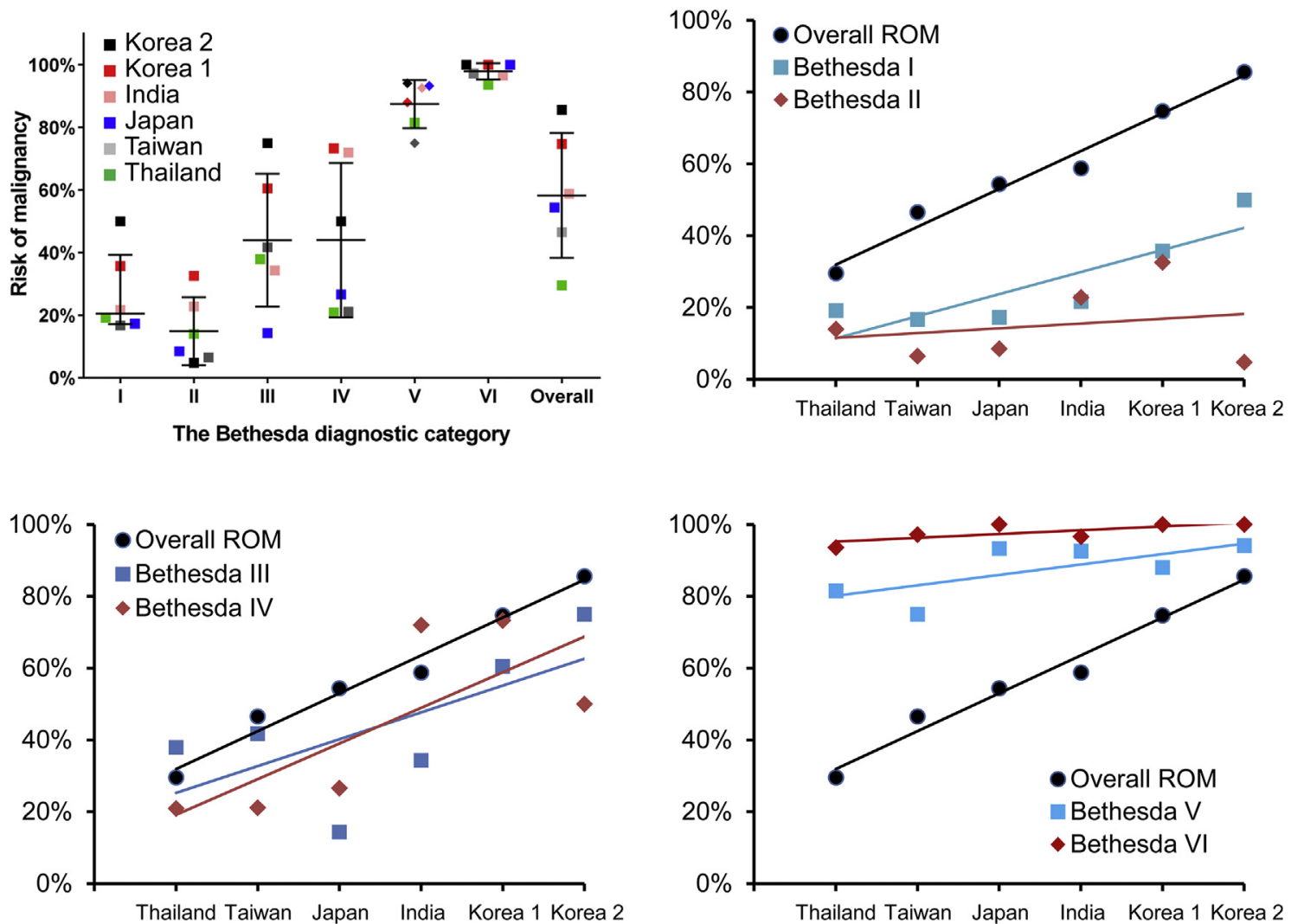

Fig. 1 Distribution of risk of malignancy (ROM) for resected nodules according to the Bethesda system category. There is a positive correlation between ROM for each Bethesda category and overall ROM among six institutions. Median and interquartile range are indicated with central horizontal and vertical lines, respectively. 
Table 3 Change of risk of malignancy in the Bethesda diagnostic categories before and after implementation of NIFTP in the individual institutions

\begin{tabular}{|c|c|c|c|c|c|c|c|}
\hline & \multicolumn{6}{|c|}{ Bethesda system diagnostic category } & \multirow[t]{2}{*}{ Total } \\
\hline & I & II & III & IV & $\mathrm{V}$ & VI & \\
\hline \multicolumn{8}{|c|}{ 1. King Chulalongkorn Memorial Hospital, Bangkok, Thailand (2010-2015), total FNA $2762^{16}$} \\
\hline FNA with surgical follow-up, $n$ & 104 & 207 & 29 & 43 & 27 & 47 & 457 \\
\hline Malignancy on surgery, $n$ & 20 & 29 & 11 & 9 & 22 & 44 & 135 \\
\hline ROM, \% & $19.2 \%$ & $14.0 \%$ & $37.9 \%$ & $20.9 \%$ & $81.5 \%$ & $93.6 \%$ & \\
\hline NIFTP, $n$ & 3 & 6 & 0 & 2 & 1 & 0 & 12 \\
\hline ROM without NIFTP, \% & $16.3 \%$ & $11.1 \%$ & $37.9 \%$ & $16.3 \%$ & $77.8 \%$ & $93.6 \%$ & \\
\hline Relative decrease in ROM, \% & $15.0 \%$ & $20.7 \%$ & $0 \%$ & $22.2 \%$ & $4.5 \%$ & $0 \%$ & \\
\hline \multicolumn{8}{|c|}{ 2. Taipei Veterans General Hospital, Taipei, Taiwan (2010-2011), total FNA 3060} \\
\hline FNA with surgical follow-up, $n$ & 30 & 155 & 24 & 19 & 16 & 143 & 387 \\
\hline Malignancy on surgery, $n$ & 5 & 10 & 10 & 4 & 12 & 139 & 180 \\
\hline ROM, \% & $16.7 \%$ & $6.5 \%$ & $41.7 \%$ & $21.1 \%$ & $75.0 \%$ & $97.2 \%$ & \\
\hline NIFTP, $n$ & 1 & 3 & 3 & 3 & 1 & 0 & 11 \\
\hline ROM without NIFTP, \% & $13.3 \%$ & $4.5 \%$ & $29.2 \%$ & $5.3 \%$ & $68.8 \%$ & $97.2 \%$ & \\
\hline Relative decrease in ROM, \% & $20.0 \%$ & $30.0 \%$ & $30.0 \%$ & $75.0 \%$ & $8.3 \%$ & $0 \%$ & \\
\hline \multicolumn{8}{|c|}{ 3. Yamashita Thyroid Hospital, Fukuoka, Japan (2015), total FNA 1229} \\
\hline Malignancy on surgery, $n$ & 9 & 6 & 5 & 17 & 28 & 158 & 223 \\
\hline $\mathrm{ROM}, \%$ & $17.3 \%$ & $8.5 \%$ & $14.3 \%$ & $26.6 \%$ & $93.3 \%$ & $100 \%$ & \\
\hline NIFTP, $n$ & 2 & 1 & 0 & 4 & 1 & 1 & 9 \\
\hline ROM without NIFTP, \% & $13.5 \%$ & $7.0 \%$ & $14.3 \%$ & $20.3 \%$ & $90.0 \%$ & $99.4 \%$ & \\
\hline Relative decrease in ROM, \% & $22.2 \%$ & $16.7 \%$ & $0.0 \%$ & $23.5 \%$ & $3.6 \%$ & $0.6 \%$ & \\
\hline \multicolumn{8}{|c|}{ 4. Gangnam Severance Hospital, Yonsei University, Seoul, South Korea (2014), total FNA 1870} \\
\hline FNA with surgical follow-up, $n$ & 28 & 43 & 76 & 15 & 25 & 145 & 332 \\
\hline Malignancy on surgery, $n$ & 10 & 14 & 46 & 11 & 22 & 145 & 248 \\
\hline $\mathrm{ROM}, \%$ & $35.7 \%$ & $32.6 \%$ & $60.5 \%$ & $73.3 \%$ & $88.0 \%$ & $100 \%$ & \\
\hline NIFTP, $n$ & 0 & 1 & 3 & 0 & 2 & 0 & 6 \\
\hline ROM without NIFTP, \% & $35.7 \%$ & $30.2 \%$ & $56.6 \%$ & $73.3 \%$ & $80.0 \%$ & $100 \%$ & \\
\hline Relative decrease in ROM, \% & $0 \%$ & $7.1 \%$ & $6.5 \%$ & $0 \%$ & $9.1 \%$ & $0 \%$ & \\
\hline \multicolumn{8}{|c|}{ 5. Seoul St. Mary's Hospital, The Catholic University, Seoul, South Korea (2014), total FNA 1112} \\
\hline FNA with surgical follow-up, $n$ & 8 & 21 & 16 & 2 & 17 & 144 & 208 \\
\hline Malignancy on surgery, $n$ & 4 & 1 & 12 & 1 & 16 & 144 & 178 \\
\hline ROM, \% & $50.0 \%$ & $4.8 \%$ & $75.0 \%$ & $50.0 \%$ & $94.1 \%$ & $100 \%$ & \\
\hline NIFTP, $n$ & 0 & 0 & 1 & 1 & 2 & 2 & 6 \\
\hline ROM without NIFTP, \% & $50.0 \%$ & $4.8 \%$ & $68.8 \%$ & $0 \%$ & $82.4 \%$ & $98.6 \%$ & \\
\hline FNA with surgical follow-up, $n$ & 23 & 57 & 35 & 50 & 27 & 58 & 250 \\
\hline Malignancy on surgery, $n$ & 5 & 13 & 12 & 36 & 25 & 56 & 147 \\
\hline ROM, \% & $21.7 \%$ & $22.8 \%$ & $34.3 \%$ & $72.0 \%$ & $92.6 \%$ & $96.6 \%$ & \\
\hline NIFTP, $n$ & 0 & 0 & 6 & 9 & 0 & 0 & 15 \\
\hline ROM without NIFTP, \% & $21.7 \%$ & $22.8 \%$ & $17.1 \%$ & $54.0 \%$ & $92.6 \%$ & $96.6 \%$ & \\
\hline Relative decrease in ROM, \% & $0 \%$ & $0 \%$ & $50.0 \%$ & $25.0 \%$ & $0 \%$ & $0 \%$ & \\
\hline
\end{tabular}

FNA, fine-needle aspiration; NIFTP, non-invasive follicular thyroid neoplasm with papillary-like nuclear features; ROM, risk of malignancy.

FN/SFN category showed a marginally significant decrease in risk of malignancy after implementation of NIFTP ( $p=0.06$, Fisher's exact test).

\section{Invasive eFV-PTC versus NIFTP}

Our cohort included a comparable number of invasive eFV-PTC $(n=48)$, which by definition had essentially similar morphology to NIFTP excluding capsular or vascular invasion. These features can be recognised on histopathological examination, but not on cytology. More than half of the cases of NIFTP $(54.2 \%)$ and invasive eFVPTC $(58.3 \%)$ were classified within indeterminate categories III and IV (Table 4). The next most frequent category for NIFTP was benign, and for invasive eFV-PTC was suspicious for malignancy. Invasive eFV-PTC demonstrated the higher prevalence of categories $\mathrm{V}-\mathrm{VI}$ compared to NIFTP ( $p=0.04$, Fisher's exact test), while NIFTP was more frequently classified as benign on FNA $(p=0.05$, Fisher's exact test).

\section{DISCUSSION}

This is the largest study to date based on the cohort of 11,372 thyroid FNA samples from three geographically distinct parts of Asia, including Eastern (Japan, Korea, Taiwan), Southern (India), and Southeastern (Thailand). We found a low incidence of NIFTP among all excised thyroid nodules $(2.9 \%)$ and among all malignant nodules $(5.3 \%)$, which is consistent with our previous report. ${ }^{15}$ The majority of NIFTP cases were classified in indeterminate categories, similarly to invasive eFV-PTC. However, despite a substantial overlap between NIFTP and invasive eFV-PTC, there was a significantly higher prevalence of benign cytology in NIFTP and SM/ malignant in invasive eFV-PTC. The most striking decrease in risk of malignancy was observed for the FN/SFN category; however, the magnitude of decrease for most categories was less than reported recently.

Consistently with previous studies, we found that most of the NIFTP cases were clustered within Bethesda categories III, IV and V. ${ }^{7-12}$ A substantial proportion of NIFTP with 
Table 4 Distribution of NIFTP versus invasive eFV-PTC according to the Bethesda system categories

\begin{tabular}{|c|c|c|c|c|c|c|c|}
\hline & \multicolumn{6}{|c|}{ Bethesda system diagnostic category } & \multirow[t]{2}{*}{ Total } \\
\hline & I & II & III & IV & $\mathrm{V}$ & VI & \\
\hline NIFTP, $n(\%)$ & $6(10.2 \%)$ & $11(18.6 \%)$ & $13(22.0 \%)$ & $19(32.2 \%)$ & $7(11.9 \%)$ & $3(5.1 \%)$ & $59(100 \%)$ \\
\hline inv-eFVPTC, $n(\%)$ & $1(2.1 \%)$ & $3(6.3 \%)$ & $16(33.3 \%)$ & $12(25.0 \%)$ & $9(18.8 \%)$ & $7(14.6 \%)$ & $48(100 \%)$ \\
\hline
\end{tabular}

inv-eFVPTC invasive encapsulated follicular variant of papillary thyroid carcinoma; NIFTP, non-invasive follicular thyroid neoplasm with papillary-like nuclear features.

non-diagnostic (14\%), benign (15-17\%) and malignant $(9-13 \%)$ cytology has been reported recently. ${ }^{7,10,12,18}$ In our study, $33.9 \%$ NIFTP were out of the indeterminate categories and occupied benign, malignant, and non-diagnostic groups, which implies that this tumour can be preceded by any of the Bethesda categories.

In line with these observations, malignancy risk after adjustment to NIFTP was affected in each category, and the greatest impact was for FN/SFN. A comparison of our findings with previous studies ${ }^{7,10-12}$ is shown in Fig. 2. The recent meta-analysis showed that NIFTP remarkably affected risk of malignancy for indeterminate diagnostic categories as a collective group. ${ }^{11}$ However, a significant variation in the extent of reduction was noted among the studies. Two studies reported the highest reduction in the risk of malignancy for FN/SFN, ${ }^{7,10}$ and another two for the benign category. ${ }^{11,12}$ The latter impact is rarely addressed in the context of NIFTP reclassification. NIFTP is considered as an equivalent of carcinoma in situ with a propensity to progress to invasive eFV-PTC. ${ }^{5}$ We believe that special attention should be given to the subset of NIFTP with benign cytology, which may require closer monitoring compared to non-neoplastic benign nodules. On the other hand, there was a low impact for the malignant category, which could predict a very small increase in false positives. Previous studies consistently reported a high impact for the SM diagnostic category, with a relative decrease in the risk of malignancy ranging from $21 \%$ to $48 \%$. $^{7,10-12}$ It may have significant translational benefit because patients with thyroid nodules reported as SM on FNA are usually subjected to total or near-total thyroidectomy, which is considered as overtreatment for NIFTP. ${ }^{6}$ However,

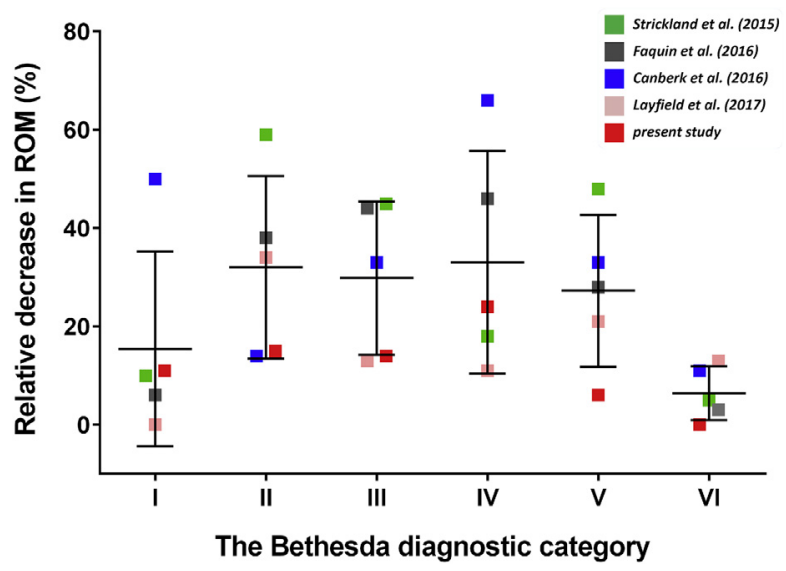

Fig. 2 Summary of published data on the relative decrease in risk of malignancy (ROM) for the Bethesda diagnostic categories after implementation of NIFTP. Median and interquartile range are indicated with central horizontal and vertical lines, respectively. the impact in the SM category in the present study was low, with only $5.6 \%$ relative reduction in risk of malignancy. Furthermore, the relative decrease in risk of malignancy in our series was substantially lower than in Western practice (Fig. 2), which is attributed to the low incidence of NIFTP.

The accurate worldwide rate of NIFTP remains to be established. ${ }^{6}$ Initial estimates of Nikiforov et al. (18.6\% of all PTC) were confirmed by several American and European institutions soon after. $5,7,12,13$ However, the most recent publications from the USA, Canada, Switzerland, the UK, and Korea have reported a much lower incidence of NIFTP, ranging from $1.5 \%$ to $6.3 \% .^{19-24}$ Our group retrospectively analysed 26,604 surgically excised PTC from the nine institutions representing Japan, Korea, China, Taiwan, Thailand, and Vietnam, and found that NIFTP rate was more than 10 times rarer $(1.5 \%)$ compared with the original series of Nikiforov et al. ${ }^{5,15}$ This trend was also observed for FVPTC. ${ }^{15}$ In the present study, we extended our findings to one more major Asian country, India, which also showed a low incidence of NIFTP.

The reasons behind this discrepancy between Western and Asian series are likely multifactorial. Despite the wellestablished diagnostic criteria, there is a high rate of disagreement among expert pathologists regarding the evaluation of PTC nuclei and other features of FV-PTC; particularly, a low agreement was found between American and Japanese experts. ${ }^{25}$ One recent report showed that Korean pathologists have a higher threshold to recognise PTC nuclei compared to their US colleagues. ${ }^{26}$ Another reason for the different incidence of FV-PTC is that geographic and ethnic (genetic traits, diet) background may influence the distinct mutation profile and molecular mechanisms of Asian PTC. ${ }^{27,28}$ There are also several factors related to the data collection which may affect NIFTP rate, such as retrospective design, primary search via surgical pathology or cytopathology database, inclusion or exclusion of microcarcinomas.

The authors of previous studies on NIFTP impact emphasised that their findings were specific for individual institutions. ${ }^{7,12}$ All four preceding reports showed a significant impact of non-invasive eFV-PTC reclassification on the risk of malignancy, which corroborated the high institutional rate of NIFTP. ${ }^{7,10-12}$ At variance, a low incidence of NIFTP in our Asian cohort translated to a lower impact of this entity on cytological and, presumably, clinical practice. The present study is also a good example to confirm that NIFTP impact needs to be evaluated for an individual institution, in the same manner as it was advised for TBSRTC. ${ }^{1}$ All participating hospitals established baseline characteristics of their thyroid FNA practice, including workload, distribution of the Bethesda categories, resection rates, risk of malignancy, the incidence of NIFTP, and risk of malignancy after NIFTP 
adjustment (Table 3). These output data from Asian countries may serve as a reference for future NIFTP studies and metaanalysis of TBSRTC.

There was a variation among individual institutions regarding characteristics of the patients (mean age, sex ratio), distribution of the surgically excised nodules according to TBSRTC, and risk of malignancy for each Bethesda category (Table 3). Such variation is attributed to the demographics, different prevalence of thyroid cancer, and different practice patterns, including admission requirements for patients, the diagnostic workflow of thyroid nodules, indications to surgery, cytological diagnostic criteria, and referral criteria. Nevertheless, a low rate of NIFTP was consistently observed in all the institutions, ranging from $1.8-6.0 \%$ among all excised nodules, and $2.4-10.2 \%$ of all malignancies. The impact of NIFTP reclassification on the Bethesda diagnostic categories at individual institutions reproduced a general trend. In most of the institutions, the greatest relative decrease in risk of malignancy was observed for FN/SFN, followed by AUS/FLUS, and benign categories (Table 2). Variation was associated with the low or high prevalence of a certain category (e.g., benign) in the institution.

NIFTP is an immediate precursor of invasive eFV-PTC. ${ }^{5}$ Both tumours are essentially the same microscopically, except for presence of capsular or angioinvasion, which renders a diagnosis of invasive eFV-PTC. FNA cytology cannot definitely distinguish NIFTP from invasive eFV-PTC due to shared microfollicular architecture and nuclear features of PTC. However, these tumours have a different prognosis and require a different treatment strategy, which can result in clinicians putting additional pressure on cytopathologists to render a preoperative diagnosis of NIFTP or invasive eFV-PTC. Several studies found a significant cytological overlap between NIFTP and invasive eFV-PTC, which were frequently associated with AUS and FN/SFN diagnostic categories. ${ }^{9,18,29}$ Our series showed a left-sided shift towards benign for NIFTP versus a right-sided shift towards malignant for invasive eFV-PTC on the Bethesda categories scale (Table 4). It is in line with the stepwise concept of development of follicular-patterned thyroid tumours; however, definite distinctive cytological features are not established. Some authors have proposed nuclear score to help differentiate invasive versus non-invasive tumours. ${ }^{9,29}$ Nevertheless, at this time there are no reliable criteria to accurately distinguish NIFTP and eFV-PTC on FNA. Refinement of cytological criteria to accommodate NIFTP in the lower risk TBSRTC categories is currently debated. ${ }^{17,30}$

Molecular testing can be considered as a promising ancillary approach to enhance the results of preoperative FNA. There is a continuing search for NIFTP-specific molecular signatures. ${ }^{29}$ It should be noted that introduction of NIFTP as a non-malignant neoplasm changed the performance of the currently available commercial molecular tests. It was reported that the positive predictive value of gene expression classifier Afirma and mutation/fusion panel ThyroSeq v2 in indeterminate thyroid nodules significantly decreased after adjustment to NIFTP. ${ }^{31,32}$ These effects, again, were dependent on the prevalence of NIFTP in a given population. Our study suggests that the impact of NIFTP on the performance of molecular tests in Asian practice will be low, compared to the Western series.

In conclusion, our large-scale multi-institutional study from five Asian countries found a low incidence of NIFTP compared to the Western series. The majority of NIFTP cases were classified in indeterminate categories, which decreased the corresponding risk of malignancy. However, the extent of NIFTP impact in our settings was much lower than in the Western reports. Asian countries may not experience significant effect of NIFTP reclassification on the practice of thyroid cytopathology, including altered risk of malignancy, refinement of cytological criteria, and performance of molecular tests. Individual institution rate of NIFTP directly predicts the impact of this entity on the current practices and necessity of related adjustments. More studies from Asian countries are highly anticipated to extend our findings.

Conflicts of interest and sources of funding: This study was supported by the Rachadapisek Sompot Fund for Postdoctoral Fellowship, Chulalongkorn University, Bangkok, Thailand to AB; a grant (NRF-2017R1D1A1B03029597) of Basic Science Research Program through the National Research Foundation of Korea funded by the Ministry of Science, ICT and Future Planning; and a grant (HI16C2013) of the Korean Health Technology R\&D Project, Ministry of Health \& Welfare, Republic of Korea. The authors state that there are no conflicts of interest to disclose.

Address for correspondence: Chan Kwon Jung, MD, $\mathrm{PhD}$, Department of Hospital Pathology, Seoul St Mary's Hospital, College of Medicine, The Catholic University of Korea, 222 Banpo-daero, Seocho-gu, Seoul 06591, Republic of Korea. E-mail: ckjung@catholic.ac.kr

\section{References}

1. Cibas ES, Ali SZ. The Bethesda system for reporting thyroid cytopathology. Am J Clin Pathol 2009; 132: 658-65.

2. Bongiovanni M, Spitale A, Faquin WC, et al. The Bethesda system for reporting thyroid cytopathology: a meta-analysis. Acta Cytol 2012; 56 $333-9$.

3. Sheffield BS, Masoudi H, Walker B, et al. Preoperative diagnosis of thyroid nodules using the Bethesda System for Reporting Thyroid Cytopathology: a comprehensive review and meta-analysis. Expert Rev Endocrinol Metab 2014; 9: 97-110.

4. Straccia P, Rossi ED, Bizzarro T, et al. A meta-analytic review of the Bethesda System for Reporting Thyroid Cytopathology: has the rate of malignancy in indeterminate lesions been underestimated? Cancer Cytopathol 2015; 123: 713-22.

5. Nikiforov YE, Seethala RR, Tallini G, et al. Nomenclature revision for encapsulated follicular variant of papillary thyroid carcinoma: a paradigm shift to reduce overtreatment of indolent tumours. JAMA Oncol 2016; 2: 1023-9.

6. Haugen BR, Sawka AM, Alexander EK, et al. American Thyroid Association Guidelines on the Management of Thyroid Nodules and Differentiated Thyroid Cancer Task Force review and recommendation on the proposed renaming of encapsulated follicular variant papillary thyroid carcinoma without invasion to noninvasive follicular thyroid neoplasm with papillary-like nuclear features. Thyroid 2017; 27: $481-3$.

7. Faquin WC, Wong LQ, Afrogheh AH, et al. Impact of reclassifying noninvasive follicular variant of papillary thyroid carcinoma on the risk of malignancy in the Bethesda System for Reporting Thyroid Cytopathology. Cancer Cytopathol 2016; 124: 181-7.

8. Howitt BE, Chang S, Eszlinger M, et al. Fine-needle aspiration diagnoses of noninvasive follicular variant of papillary thyroid carcinoma. Am J Clin Pathol 2015; 144: 850-7.

9. Maletta F, Massa F, Torregrossa L, et al. Cytological features of "noninvasive follicular thyroid neoplasm with papillary-like nuclear features" and their correlation with tumour histology. Hum Pathol 2016; 54: $134-42$

10. Canberk S, Gunes P, Onenerk M, et al. New concept of the encapsulated follicular variant of papillary thyroid carcinoma and its impact on the Bethesda System for Reporting Thyroid Cytopathology: a singleinstitute experience. Acta Cytol 2016; 60: 198-204.

11. Layfield LJ, Baloch ZW, Esebua M, et al. Impact of the reclassification of the non-invasive follicular variant of papillary carcinoma as benign on 
the malignancy risk of the Bethesda System for Reporting Thyroid Cytopathology: a meta-analysis study. Acta Cytol 2017; 61: 187-93.

12. Strickland KC, Howitt BE, Marqusee E, et al. The impact of noninvasive follicular variant of papillary thyroid carcinoma on rates of malignancy for fine-needle aspiration diagnostic categories. Thyroid 2015; 25 987-92.

13. Rosario PW, Mourao GF, Nunes MB, et al. Noninvasive follicular thyroid neoplasm with papillary-like nuclear features. Endocr Relat Cancer 2016; 23: 893-7.

14. Ferlay J, Soerjomataram I, Ervik M, et al. GLOBOCAN 2012 v1.0, cancer incidence and mortality worldwide: IARC CancerBase No. 11 Lyon, France: International Agency for Research on Cancer, 2013.

15. Bychkov A, Hirokawa M, Jung CK, et al. Low rate of noninvasive follicular thyroid neoplasm with papillary-like nuclear features in Asian practice. Thyroid 2017; 27: 983-4.

16. Limlunjakorn P, Keelawat S, Bychkov A. Evaluation of thyroid fine needle aspiration cytology by the Bethesda reporting system: a retrospective analysis of rates and outcomes from the King Chulalongkorn Memorial Hospital. J Med Assoc Thai 2017; 100: 783-92.

17. Pusztaszeri M, Rossi ED, Auger M, et al. The Bethesda System for Reporting Thyroid Cytopathology: proposed modifications and updates for the second edition from an international panel. Acta Cytol 2016; 60: 399-405.

18. Ibrahim AA, Wu HH. Fine-needle aspiration cytology of noninvasive follicular variant of papillary thyroid carcinoma is cytomorphologically distinct from the invasive counterpart. Am J Clin Pathol 2016; 146 : $373-7$.

19. Golding A, Shively D, Bimston DN, et al. Noninvasive encapsulated follicular variant of papillary thyroid cancer: clinical lessons from a community-based endocrine surgical practice. Int J Surg Oncol 2017; 2017: 4689465.

20. Cho U, Mete O, Kim MH, et al. Molecular correlates and rate of lymph node metastasis of non-invasive follicular thyroid neoplasm with papillary-like nuclear features and invasive follicular variant papillary thyroid carcinoma: the impact of rigid criteria to distinguish noninvasive follicular thyroid neoplasm with papillary-like nuclear features. Mod Pathol 2017; 30: 810-25.
21. Parente DN, Kluijfhout WP, Bongers PJ, et al. Clinical safety of renaming encapsulated follicular variant of papillary thyroid carcinoma: is NIFTP truly benign? World J Surg 2018; 42: 321-6.

22. Lee SE, Hwang TS, Choi YL, et al. Molecular profiling of papillary thyroid carcinoma in Korea with a high prevalence of BRAFV600E mutation. Thyroid 2017; 27: 802-10.

23. Poller DN, Johnson SJ, Stephenson TJ. Diagnosis of NIFTP in the UK. J Basic Clin Med 2017; 6: 63-4.

24. Saglietti C, Bongiovanni M. The value of cytological examination in the diagnosis of noninvasive thyroid neoplasm with papillary-like nuclear features (NIFTP). J Basic Clin Med 2017; 6: 57-60.

25. Hirokawa M, Carney JA, Goellner JR, et al. Observer variation of encapsulated follicular lesions of the thyroid gland. Am J Surg Pathol 2002; 26: $1508-14$

26. Jung CK, Kim C. Effect of lowering the diagnostic threshold for encapsulated follicular variant of papillary thyroid carcinoma on the prevalence of non-invasive follicular thyroid neoplasm with papillarylike nuclear features: a single-institution experience in Korea. $J$ Basic Clin Med 2017; 6: 26-8.

27. Bychkov A. Prevalence of BRAFV600E mutation in Asian patients with thyroid cancer. Malays J Pathol 2017; 39: 95-6.

28. Song YS, Lim JA, Park YJ. Mutation profile of well-differentiated thyroid cancer in Asians. Endocrinol Metab (Seoul) 2015; 30: 252-62.

29. Zhao L, Dias-Santagata D, Sadow PM, et al. Cytological, molecular, and clinical features of noninvasive follicular thyroid neoplasm with papillary-like nuclear features versus invasive forms of follicular variant of papillary thyroid carcinoma. Cancer Cytopathol 2017; 125: 323-31.

30. LiVolsi VA, Layfield L, Baloch ZW. Non-invasive follicular neoplasm with papillary like nuclear features (NIFTP): if it ain't broke, don't fix it. The cytopathologist's dilemma. Diagn Cytopathol 2017; 45: 479-80.

31. Hang JF, Westra WH, Cooper DS, et al. The impact of noninvasive follicular thyroid neoplasm with papillary-like nuclear features on the performance of the Afirma gene expression classifier. Cancer Cytopathol 2017: 125: 683-91.

32. Valderrabano P, Khazai L, Leon ME, et al. Evaluation of ThyroSeq v2 performance in thyroid nodules with indeterminate cytology. Endocr Relat Cancer 2017; 24: 127-36. 